\title{
Correlation of Low HDL and High Triglycerides with Outcome in Patients with COVID 19 Infection
}

\author{
Sanjay Tukaram Thorat ${ }^{1}$, Vaibhav Vijay Rane ${ }^{2}$, Radhika Bajaj ${ }^{3}$, Avanti Damle ${ }^{4}$, Nikhil Patil ${ }^{5}$, Pankaj Pawar ${ }^{6}$ \\ 1,2,3,4,5,6Department of Medicine, Krishna Institute of Medical Sciences, Karad, Maharashtra, India.
}

\section{ABSTRACT}

\section{BACKGROUND}

The world is still experiencing corona virus disease-19 (COVID-19) pandemic. So far, we experienced a total of more than 23 crore cases and 47 lakh deaths from COVID 19 disease. Severe acute respiratory syndrome - corona virus - 2 (SARSCoV-2) was believed to affect lipid metabolism, with many authors reporting an increase in triglycerides and a decrease in high density lipoprotein (HDL) levels. This study gave the clinical features of COVID-19 patients with various HDL-C levels and an interrelation between HDL-C levels and the risk for adverse outcome in the form of deaths.

\section{METHODS}

We conducted a cross sectional study on 100 COVID-19 adult patients diagnosed by reverse transcription - polymerase chain reaction (RT-PCR) test admitted to the medicine department, from January 2020 to December 2020, who were also tested for lipid parameters. The detailed history and lab parameters of the patients were collected and the severe outcome of the same was measured in terms of deaths.

\section{RESULTS}

The mean age of study participants was $57.92 \pm 12.41$ years. Majority of the participants were from the age group of 41 to 60 years with 50 patients (50\%). There were 73 males (73\%) and 27 females (27\%) in our study. We observed that a total of 36 patients had co-morbidities ( $36 \%$ ), such as diabetes seen in 22 cases (22\%), hypertension in 18 cases (18\%), ischaemic heart disease (IHD) in 8 cases (8 $\%)$. A significant association was seen between the presence of co-morbidities and deaths in our study $(P=0.043)$. A significant association was seen between the patients who required intensive care and deaths $(P<0.001)$. We found a significant difference between the triglycerides and HDL parameters of lipid profiles in patients who died as compared to those who survived. $(P<0.05)$ The mean triglyceride level in patients who died was $223.14 \pm 56.59$, significantly higher than those who survived $134.43 \pm 96.16$. $(\mathrm{P}=0.003)$

\section{CONCLUSIONS}

The lipid profile evaluation in our study was found to be effective in detecting the correlation of severity and outcome in COVID-19 patients. We conclude that the severity of COVID-19 cases is associated with low HDL and high triglyceride levels.
Corresponding Author: Dr. Vaibhav Vijay Rane, Department of Medicine, Krishna Institute of Medical Sciences, Karad, Maharashtra, India.

E-mail: www.vaibhavrane.vr@gmail.com

DOI: $10.14260 /$ jemds/2021/784

How to Cite This Article:

Thorat ST, Rane VV, Bajaj R, et al. Correlation of low HDL and high triglycerides with outcome in patients with COVID 19 infection. J Evolution Med Dent Sci 2021;10(45):3880-3883, DOI: $10.14260 / j e m d s / 2021 / 784$

Submission 08-11-2021,

Peer Review 06-12-2021,

Acceptance 13-12-2021,

Published 23-12-2021.

Copyright (c) 2021 Sanjay Tukaram Thorat et al. This is an open access article distributed under Creative Commons Attribution License [Attribution 4.0 International (CC BY 4.0)]

\section{KEY WORDS}

COVID-19, Lipid Profile, HDL, Triglycerides, Patient Outcome. 


\section{BACKGROUND}

COVID 19 infection caused by severe acute respiratory syndrome corona virus - 2, which was first reported in Wuhan in 2019, has spread globally. ${ }^{1,2}$ As of September 2021, the world has experienced a total of more than 23 crore cases and 47 lakh deaths from COVID-19 disease. ${ }^{3,4}$ India reported more than 3 crore cases and around 4.4 lakh deaths from COVID-19 disease. 5 The pandemic is not yet over and all over the world healthcare facilities are struggling to handle the cases of COVID-19 disease.

As the number of COVID-19 patients is dramatically increasing worldwide and treatment in intensive care units (ICU) has become a major challenge, early recognition of severe forms of COVID-19 is essential for timely triaging patients. ${ }^{3}$ While the clinical status, in particular peripheral oxygen saturation levels and concurrent co-morbidities of COVID-19 patients, several laboratory parameters may facilitate the assessment of disease severity. Parameters like serum levels of C-reactive protein (CRP), D-dimers ferritin, and lipid profile may be used in the risk stratification to predict severity in COVID-19 in hospitalized patients. ${ }^{3}$

SARS-CoV-2 was believed to affect lipid metabolism. The lipid profile parameters significantly changed in COVID-19 patients with many authors reporting an increase in triglycerides and a decrease in HDL in patients with COVID19 disease, but the variation and effects of high-density lipoprotein cholesterol (HDL-C) in COVID-19 were uncommon. High-density lipoproteins (HDLs) transport cholesterol from extra hepatic tissues to the liver for its metabolism. Even though the primary role of HDLs is the prevention of atherosclerosis, many recent studies have found different properties of HDLs like anti-infection, antiinflammatory, anti-apoptotic or antioxidant properties. 6,7

In addition, HDLs are believed to protect against many infectious diseases. It was noticed that patients with low HDL levels manifested as having an increased risk of infection and a poor outcome. ${ }^{8,9}$ Hence, it was concluded that HDL levels are perhaps associated with the outcome of COVID-19 patients. This study gave the clinical features of COVID-19 patients with various HDL-C levels and an interrelation between HDL$\mathrm{C}$ levels and the risk for developing severe events.

\section{Objectives}

- To assess the lipid profile in patients with COVID-19.

- To compare HDL and triglycerides with severity in COVID-19 infection.

\section{METHODS}

This was a cross sectional study done at the Department of Medicine at Krishna Medical College and Hospital, Karad, A tertiary care centre and teaching hospital in Western Maharashtra.

All adult COVID-19 patients diagnosed by reverse transcription-polymerase chain reaction admitted to the medicine department, from January 2020 to December 2020, who were tested for blood lipid levels were considered in the current study. Patients with age below 18 years and those who had not been tested for blood lipids were excluded. Ethical approval was taken from the Institutional Ethics Committee before starting the study. COVID-19 positive patients were enrolled, detailed history and lab parameters were collected, the severe outcome was measured in terms of deaths.

\section{Sample Size Calculation}

According to study conducted by Wang G et al.10 the patients with low HDL cholesterol had $37 \%$ severe cases, so $p=37 \%$.

Using formula for sample size (n) calculation,

$\frac{n=4 \times \mathrm{p} \times \mathrm{q}}{e^{2}}$

Where,

$\mathrm{p}=37 \%=0.37$

$\mathrm{q}=1-\mathrm{p}=0.63$

Taking e, absolute error of $10 \%, \mathrm{e}=0.1$

So,

$\frac{n=4 \times 0.37 \times 0.63}{0.1 \times 0.1}$

$\mathrm{N}=93.24=94$

A minimum of 94 patients was to be included in the study, rounding it up to 100 .

\section{Statistical Analysis}

Data was collected using a semi-structured pretested questionnaire; data collected was entered in Microsoft Excel. Data is represented in frequencies, percentages, charts and graphs. The mean and standard deviation of quantitative variables are shown. Appropriate statistical tests are applied using Statistical Package for Social Sciences (SPSS) software version 21 for analysis. The chi-square test is used for association and the student's t-test is used for comparison between the study variables. Statistical significance is considered at $\mathrm{p}<0.05$.

\section{RESULTS}

The mean age of study participants was $57.92 \pm 12.41$ years. The majority of the participants were from the age group of 41 to 60 years with 50 patients ( $50 \%$ ) followed by 61 to 80 years with 32 patients (32\%) and 21 to 40 years with 18 participants $(18 \%)$.

No significant difference was seen between the mean age of the participants who survived and died in our study ( $\mathrm{p}=$ 0.46). The mean age of patients who survived was $57.71 \pm$ 10.24 years and the patients who died was $58.67 \pm 13.44$ years.

There were 73 males (73\%) and 27 females (27\%) in our study. The male to female ratio was $2.7: 1$. There was no significant association between gender and survival $(\mathrm{p}=$ 0.71). Out of 27 females, 2 expired ( $7.41 \%)$ and 25 survived (92.59\%). Out of 73 males, 5 expired (6.85\%) and 68 expired $(93.15 \%)$. 
We observed that a total of 36 patients had comorbidities (36\%), such as diabetes seen in 22 cases (22\%), hypertension in 18 cases (18\%), IHD in 8 cases (8\%), chronic obstructive pulmonary disease (COPD) in 4 cases (4 $\%)$. A significant association was seen between the presence of co-morbidities and deaths in our study ( $\mathrm{p}=0.043)$, with 5 deaths (13.89\%) amongst 36 cases with co-morbidities and just 2 deaths (3.135) amongst 64 cases with co-morbidities.

\begin{tabular}{|cccc|}
\hline & No Co-morbidities & Co-morbidities & Total \\
\hline Survived & 62 & 31 & 93 \\
Expired & 2 & 5 & 7 \\
Total & $\mathbf{6 4}$ & $\mathbf{3 6}$ & $\mathbf{1 0 0}$ \\
\hline \multicolumn{2}{|l}{ Table 1. Association between Comorbidities and Deaths } \\
\hline $\mathrm{P}<0.001$, Significant
\end{tabular}

A significant association was seen between the patients who required intensive care and deaths $(p<0.001)$. A total of 16 cases required ICU admissions; out of which 6 had died (37.5\%) and out of 84 cases managed in the ward, only one patient had died (1.19\%). (Table 1 )

We found a significant difference in lab parameters of patients who died than those who survived in the case of aspartate aminotransferase (AST), alanine aminotransferase (ALT) and serum creatinine $(\mathrm{p}<0.05)$. (Table 2)

\begin{tabular}{|c|c|c|c|c|}
\hline \multicolumn{2}{|c|}{ Expired } & Mean & SD & P-Value \\
\hline \multirow{2}{*}{ D dimer } & Yes & 1.54 & 1.88 & \multirow{2}{*}{$>0.05$} \\
\hline & No & 0.69 & 2.03 & \\
\hline \multirow{2}{*}{ Ferritin } & Yes & 447.57 & 328.30 & \multirow{2}{*}{$>0.05$} \\
\hline & No & 354.72 & 373.50 & \\
\hline \multirow{2}{*}{ Pro-calcitonin } & Yes & 0.28 & 0.14 & \multirow{2}{*}{$>0.05$} \\
\hline & No & 0.76 & 1.15 & \\
\hline \multirow{2}{*}{ IL 6} & Yes & 41.00 & 27.71 & \multirow{2}{*}{$>0.05$} \\
\hline & No & 65.88 & 56.50 & \\
\hline \multirow{2}{*}{ Haemoglobin } & Yes & 11.79 & 2.42 & \multirow{2}{*}{$>0.05$} \\
\hline & No & 12.79 & 1.84 & \\
\hline \multirow{2}{*}{ TLC } & Yes & 7500.00 & 3052.87 & \multirow{2}{*}{$>0.05$} \\
\hline & No & 7258.06 & 3204.80 & \\
\hline \multirow{2}{*}{ Serum albumin } & Yes & 3.23 & 0.47 & \multirow{2}{*}{$>0.05$} \\
\hline & No & 3.28 & 0.38 & \\
\hline \multirow{2}{*}{ AST } & Yes & 60.14 & 15.57 & \multirow{2}{*}{$0.015^{*}$} \\
\hline & No & 43.83 & 34.41 & \\
\hline \multirow{2}{*}{ ALT } & Yes & 63.29 & 20.05 & \multirow{2}{*}{$0.025^{*}$} \\
\hline & No & 41.17 & 25.07 & \\
\hline \multirow{2}{*}{ Serum billirubin } & Yes & 0.94 & 0.20 & \multirow{2}{*}{$>0.05$} \\
\hline & No & 0.81 & 1.48 & \\
\hline \multirow{2}{*}{ Serum creatinine } & Yes & 1.31 & 0.45 & \multirow{2}{*}{$<0.001^{*}$} \\
\hline & No & 0.93 & 0.24 & \\
\hline \multicolumn{5}{|c|}{$\begin{array}{c}\text { Table 2. Difference in Lab Parameters of Patients Who Died Than } \\
\text { Those Who Survived }\end{array}$} \\
\hline
\end{tabular}

\section{Table 1. Lab Parameters and Deaths}

We found a significant difference between the triglycerides and HDL parameters of lipid profiles in patients who died as compared to those who survived $(p<0.05)$.

The mean triglyceride level in patients who died was $223.14 \pm 56.59$, significantly higher than those who survived $134.43 \pm 96.16(\mathrm{p}=0.003)$

The mean HDL levels in patients who died were $32.71 \pm$ 10.01 , significantly lower than those who survived $45.59 \pm$ $10.12(\mathrm{p}=0.002)$

The total cholesterol levels and LDL levels were also higher in patients who died as compared to those who survived, but without any significant statistical difference (p $>0.05$ ). (Table 3 )

\begin{tabular}{|ccccc|}
\hline Expired & & Mean & SD & P-Value \\
Total cholesterol & Yes & 160.14 & 67.05 & 0.585 \\
& No & 148.00 & 55.43 & \\
Triglycerides & Yes & 223.14 & 56.59 & $0.003^{*}$ \\
& No & 134.43 & 96.16 & \multirow{2}{*}{ HDL } \\
\multirow{2}{*}{ LDL } & Yes & 32.71 & 10.01 & $0.002^{*}$ \\
& No & 45.59 & 10.12 & \multirow{2}{*}{0.734} \\
\hline \multicolumn{5}{|c}{ Table 3. Lipid Profile Parameters \& Deaths } \\
\hline
\end{tabular}

\section{DISCUSSION}

This prospective study showed a significant association with lipid profile parameters and severity of COVID-19 cases. We observed that severe COVID-19 cases had significantly low HDL and high triglyceride levels $(\mathrm{p}<0.05)$.

In this study, it was seen that the triglyceride levels in patients who died were significantly higher than those who survived. The HDL levels in patients who died were significantly lower than those who survived. The total cholesterol levels and LDL levels were also higher in patients who died as compared to those who survived, but without any significant statistical significance.

Several studies done previously have described the rapid changes in the lipid profile in response to COVID-19 treatment and the progression of the disease being correlated with the further patient outcome. ${ }^{11-14}$

The mechanism for the effect of lipid profile on infections is not clearly understood. Many explanations for this have been proposed. During acute infections, development of a hyper catabolic status and the usual undernourishment might be the reason. Of interest, severe patients presented significantly higher triglyceride and low HDL-C levels, denoting that the factors associated with COVID 19 progression influence these lipid parameters. In the group of patients with a lipid profile that was determined before the infection, patients with worse outcomes were of older age and they also had many co-morbidities, which might have influenced the differences in the levels of both lipids. ${ }^{15,16}$

As shown in our study, the lipid levels assist with the management of COVID-19 patients. Physicians should watch for lipid profile alterations as one of the poor prognostic criteria, which will help the decision-making process and earlier treatment intensification for better patient outcome.

Our study showed that patients with low HDL-C levels and high triglycerides had a higher proportion of severe events. Hence, HDL-C and triglyceride levels may serve in a protective role in COVID-19 infection and that's why COVID19 patients with reduced HDL-C and high triglycerides need proper monitoring and treatment as soon as possible to improve the outcomes.

Masana L et al. ${ }^{11}$ observed that low HDL-C levels and high TGs during hospitalization are high risk markers for COVID19 patients. While in patients with pre-infection, low HDL cholesterol levels and high TG levels are also associated with the worse prognosis in COVID 19 cases. In some studies, it was seen that infected patients, especially those with sepsis, always had a significant decrease in HDL levels, but the cause for the decrease in HDL level was unknown. But, several hypotheses are thought to be possible, including a decrease in HDL production, overconsumption, or redistribution of HDL particles from intravascular to the extra vascular space.17-19 
Guyi Wang et al.10also found that hat low HDL-C was correlated with poor outcomes of adult COVID-19 patients, and provided a basis for HDL-C to predict COVID-19 prognosis.

In this study, it was seen that septic patients with low HDL-C levels showed higher mortality and other adverse clinical outcomes. Several studies have found significant mortality increase in sepsis patients with an HDL level below $25 \mathrm{mg} / \mathrm{dl}(0.65 \mathrm{mmol} / \mathrm{L})$, so the clinical features and outcome of COVID-19 patients with a HDL level above and below 25 $\mathrm{mg} / \mathrm{dl}(0.65 \mathrm{mmol} / \mathrm{L})$ was compared. The study concluded that patients with low HDL levels had a higher proportion of severe events, while further regression analysis also revealed that low HDL-C was an independent risk factor for severe events in COVID-19. Hence, HDL-C may play a protective role in COVID-19 infection.

Our study found that low HDL and high triglycerides were correlated with adverse outcomes of adult COVID-19 patients, and our study has its limitations of lower sample size and being a hospital based study, this was not an actual representing sample from the whole population of Covid-19 patients in the community. The basic lipid profile data before symptom onset was unknown to us, so it is undetermined if the decrease in HDL-C level occurred after infection with SAR-CoV-2. Many previous studies showed that HDLs decrease significantly in the early stage of sepsis, but the time from the symptom onset to the detection of HDLs is different, which may cause some bias in the analysis of the relationship between HDLs and COVID-19. The HDL-C level during the recovery period was undetected, and the correlation between the dynamic changes of HDL-C and the outcome of COVID-19 may be more valuable.

\section{CONCLUSIONS}

Covid-19 cases are still increasing all over the world, especially with the new serological variants and changing disease pattern in terms of symptoms and overall disease severity. It is very important to develop prognostic indicators for COVID-19 patients. The lipid profile evaluation in our study was found to be effective in detecting the correlation of severity and outcome in COVID-19 patients. We conclude that the severity of COVID-19 cases is associated with low HDL and high triglyceride levels. It is important to check these lab parameters for better patient care and suspecting the severe cases of COVID-19 to plan for better patient management.

Data sharing statement provided by the authors is available with the full text of this article at jemds.com.

Financial or other competing interests: None.

Disclosure forms provided by the authors are available with the full text of this article at jemds.com.

\section{REFERENCES}

[1] Lu H, Stratton CW, Tang YW. Outbreak of pneumonia of unknown etiology in Wuhan, China: the mystery and the miracle. J Med Virol 2020;92(4):401-2.
[2] Hui DS, Azhar EI, Madani TA, et al. The continuing 2019$\mathrm{nCoV}$ epidemic threat of novel coronaviruses to global health - the latest 2019 novel coronavirus outbreak in Wuhan, China. Int J Infect Dis 2020;91:264-6.

[3] Huang C, Wang Y, Li X, et al. Clinical features of patients infected with 2019 novel coronavirus in Wuhan, China. Lancet 2020;395(10223):497-506.

[4] Guan WJ, Ni ZY, Hu Y, et al. Clinical characteristics of coronavirus disease 2019 in China. N Engl J Med 2020;382:1708-20.

[5] Dwari K. COVID-19 Pandemic: a positive influence on lifestyle, economy, administration \& environment; an overview. Indian Journal of Forensic Medicine \& Toxicology 2020;14(4):6605-10.

[6] Casari I, Manfredi M, Metharom P, et al. Dissecting lipid metabolism alterations in SARS-CoV-2. Prog Lipid Res 2021;82:101092.

[7] Cao X, Yin R, Albrecht H, et al. Cholesterol: a new game player accelerating vasculopathy caused by SARS-CoV-2? American Journal of Physiology-Endocrinology and Metabolism 2020;319(1):E197-202.

[8] Brewer HB. Increasing HDL cholesterol levels. N Engl J Med 2004;350(15):1491-4.

[9] Pirillo A, Catapano AL, Norata GD. HDL in infectious diseases and sepsis. Handb Exp Pharmacol 2015:224:483-508.

[10] Wang G, Zhang Q, Zhao X, et al. Low high-density lipoprotein level is correlated with the severity of COVID-19 patients: an observational study. Lipids Health Dis 2020;19(1):204.

[11] Masana L, Correig E, Ibarretxe D, et al. Low HDL and high triglycerides predict COVID-19 severity. Scientific Reports 2021;11(1):7217.

[12] Hu X, Chen D, Wu L, et al. Low serum cholesterol level among patients with COVID-19 infection in Wenzhou, China. China (February 21, 2020). 2020 Feb 21.

[13] Hu X, Chen D, Wu L, et al. Declined serum high density lipoprotein cholesterol is associated with the severity of COVID-19 infection. Clin Chim Acta 2020;510:105-10.

[14] Feingold KR, Anawalt B, Boyce A, et al. Lipid and lipoprotein levels in patients with COVID-19 infections. Endotext [Internet] 2020 Nov 15.

[15] Sittiwet C, Simonen P, Gylling H, et al. Mortality and cholesterol metabolism in subjects aged 75 years and older: the Helsinki businessmen study. J Am Geriatr Soc 2020;68(2):281-7.

[16] Orkaby AR. The highs and lows of cholesterol: a paradox of healthy aging? 2020;68(2):236-7.

[17] Mattila KJ, Valtonen VV, Nieminen MS, et al. Role of infection as a risk factor for atherosclerosis, myocardial infarction, and stroke. Clin Infect Dis 1998;26(3):719-34.

[18] Grunfeld C, Pang MI, Doerrler W, et al. Lipids, lipoproteins, triglyceride clearance, and cytokines in human immunodeficiency virus infection and the acquired immunodeficiency syndrome. J ClinEndocrinol Metabol 1992;74(5):1045-52.

[19] Calza L, Manfredi R, Chiodo F. Hyperlipidaemia in patients with HIV-1 infection receiving highly active antiretroviral therapy: epidemiology, pathogenesis, clinical course and management. Int J Antimicrob Agents 2003;22(2):89-99. 